\title{
Rechtsmängel des Ärztestopps
}

\author{
Y. Hangartner*
}

\footnotetext{
* Der Autor, emeritierter Ordinarius für öffentliches Recht der Universität St. Gallen, ist Verfasser eines am 31. Juli 2002 der Verbindung der Schweizer Ärztinnen und Ärzte FMH erstatteten umfangreichen Gutachtens über Rechtmässigkeit und rechtliche Folgeprobleme von Art. 55a des Bundesgesetzes über die Krankenversicherung und der Verordnung des Bundesrates über die Einschränkung der Zulassung von Leistungserbringern zur Tätigkeit zu Lasten der obligatorischen Krankenpflegeversicherung. Im vorliegenden Beitrag kann - ohne Anspruch auf vertiefte Behandlung - nur auf die wichtigsten rechtlichen Aspekte der Einführung und Ausgestaltung der Bedürfnisklausel hingewiesen werden.
}

1 Hofmann D. La clause du besoin pour les médecins et la Constitution fédérale. Aktuelle Juristische Praxis/ Pratique juridique actuelle (AJP/PJA) 2003;12:789-800.

2 Essers UA, Fleiner T. Über die Unvereinbarkeit des Zulassungsstopps für Ärzte mit dem Freizügigkeitsabkommen Schweiz EU und dem schweizerischen Recht. Schweiz Ärztezeitung 2003;84(34):1749-50.

3 Hangartner Y. Der Grundsatz der Nichtdiskriminierung wegen der Staatsangehörigkeit im Freizügigkeitsabkommen der Schweiz mit der Europäischen Gemeinschaft.

AJP/PJA 2003;12:257-69.

Korrespondenz:

Prof. Dr. rer. publ. Yvo Hangartner Am Gozenberg 2

CH-9200 Gossau

\section{Problemstellung}

\section{Rechtliche Ausgangslage}

Mit Bundesgesetz vom 24. März 2000 wurde das Krankenversicherungsgesetz durch einen Art. 55a ergänzt. Er bestimmt, dass der Bundesrat für eine befristete Zeit von bis zu drei Jahren die Zulassung von Leistungserbringern zur Tätigkeit zu Lasten der obligatorischen Krankenpflegeversicherung nach den Art. 36-38 KVG von einem Bedürfnis abhängig machen kann. Der Bundesrat legt die entsprechenden Kriterien fest. Die Kantone bestimmen die Leistungserbringer.

Gestützt darauf erliess der Bundesrat am 3. Juli 2002 die Verordnung über die Einschränkung der Zulassung von Leistungserbringern zur Tätigkeit zu Lasten der obligatorischen Krankenpflegeversicherung. Die Zahl der Leistungserbringer wird auf eine im Anhang festgelegte Höchstzahl beschränkt. Die Kantone können vorsehen, dass die Höchstzahl nicht gilt oder, unter bestimmten Voraussetzungen, dass neue Zulassungen nicht erteilt werden; sie können aber auch zusätzliche Leistungserbringer bewilligen, wenn in der Kategorie eine Unterversorgung besteht. Die Verordnung gilt bis zum 3. Juli 2005. Ob Art. 55a KVG erlaubt, die Aktion zu verlängern bzw. zu wiederholen, blieb im Parlament unklar.

\section{Rechtsfragen}

Die rechtliche Problematik dieses sogenannten Ärztestopps (und Stopps anderer Leistungserbringer in der obligatorischen Krankenpflegeversicherung) [1] betrifft einerseits den Grundsatz in Art. 55a KVG und anderseits dessen Durchführung durch den Bundesrat in der Verordnung vom 3. Juli 2002. In der Beurteilung ist zu berücksichtigen, ob die Regelung allenfalls das Freizügigkeitsabkommen zwischen der Schweiz und der Europäischen Gemeinschaft und deren Mitgliedstaaten verletzt [2]. In den Kantonen stellt sich die Frage, ob deren Ausführungsregelungen von der kantonalrechtlich zuständigen Instanz im kantonalrechtlich richtigen Verfahren erlassen wurden.

Unerheblich für die rechtliche Beurteilung ist ein allfälliges jahrelanges Versagen der zuständigen Behörden in der Gesundheitspolitik. Die Rechtsetzung hat die öffentlichen Interessen nach den Bedürfnissen von hier und heute $\mathrm{zu}$ wahren.

\section{Verknüpfung materiell- und prozess- rechtlicher Fragen}

In der Beurteilung der Regelungen über den Ärztestopp sind materiell- und prozessrechtliche Fragen auseinanderzuhalten. Die politische Diskussion bezieht sich auf die gesamte materiellrechtliche Problematik. Beschwerden im Einzelfall sind im Rahmen der prozessualen Möglichkeiten vorzutragen.

Prozessrechtlich wird die Auseinandersetzung für betroffene Ärzte erschwert durch die Regel von Art. 191 BV, wonach Bundesgesetze für die rechtsanwendenden Behörden massgebend sind. Bundesgesetze sind anzuwenden, auch wenn sie verfassungswidrig sein sollten. Das Massgeblichkeitsgebot von Art. 191 BV entbindet aber nicht von der Verpflichtung, Bestimmungen im Rahmen von Auslegungsspielräumen verfassungskonform auszulegen. In Fragen, die das Gesetz nicht eindeutig beantwortet, ist jene Interpretation als richtig anzusehen, die der Bundesverfassung entspricht bzw. am besten entspricht. Diese Feststellung ist im vorliegenden Zusammenhang von besonderer Bedeutung, weil Art. 55a KVG die Bedürfnisklausel nicht vorschreibt, sondern ihre Einführung und Ausgestaltung in einer nicht präzise umschriebenen Ermächtigung dem Bundesrat überlässt.

\section{Grundsätzliche Problematik der (befristeten) Bedürfnisklausel}

\section{Grundrechtlicher Schutz der privat- wirtschaftlichen Erwerbstätigkeit}

Die privatwirtschaftlich ausgeübte Erwerbstätigkeit als Arzt ist durch das Grundrecht der Wirtschaftsfreiheit (Art. $27 \mathrm{BV}$ ) verfassungsrechtlich gewährleistet. Das Grundrecht schützt ausdrücklich namentlich den freien Zugang zur Erwerbstätigkeit. Auf die Wirtschaftsfreiheit können sich Schweizer Bürger und niedergelassene Ausländer berufen. Angehörige von Mitgliedstaaten der EG können aufgrund des Freizügigkeitsabkommens die ärztliche Tätigkeit in der Schweiz aufnehmen. In der Behandlung der EG-Angehörigen ist der Grundsatz der Nichtdiskriminierung zu beachten [3]. 
Verfassungsrechtlicher Schutz auch im Rahmen der obligatorischen Krankenpflegeversicherung

Den Schutz der Wirtschaftsfreiheit geniesst auch die privatwirtschaftliche ärztliche Tätigkeit zu Lasten der obligatorischen Krankenpflegeversicherung. Die Krankenpflege ist nicht verstaatlicht. Das Bundesamt für Sozialversicherung beruft sich allerdings auf den Entscheid BGE $122 \mathrm{~V} \mathrm{85}$, aus dem hervorgehe, dass sich ein Leistungserbringer, der im Rahmen der obligatorischen Krankenpflegeversicherung tätig sein will, nicht auf die Wirtschaftsfreiheit berufen und daraus einen Anspruch auf Zulassung zur Kassenpraxis ableiten könne. Die Begründung des Urteils zeigt jedoch, dass das Eidgenössische Versicherungsgericht in jenem Fall unter Berufung auf das Massgeblichkeitsgebot argumentiert hat; in bezug auf Verordnungsrecht anerkennt es ausdrücklich die Wirksamkeit der Wirtschaftsfreiheit.

Durch die neue Bundesverfassung vom 18. April 1999 ist die Wirtschaftsfreiheit überdies institutionell geschützt (Art. 94 BV). Dieser Schutz ist in der Gesetzgebung zu berücksichtigen, also auch in der Gesetzgebung über die Krankenversicherung, obwohl deren Verfassungsgrundlage (Art. 117 BV) dem Gesetzgeber einen grossen Gestaltungsspielraum einräumt. Die Regelungen sind aber mit Rücksicht auf Art. 94 BV systemkonform (rechtsgleiche Behandlung der Ärzte, Wettbewerb unter ihnen) auszugestalten.

Die Frage der Geltung der Wirtschaftsfreiheit sollte im vorliegenden Zusammenhang allerdings nicht überschätzt werden. Art. 55a KVG und die Verordnung des Bundesrates dazu verstossen nicht grundsätzlich, von vornherein, gegen die Wirtschaftsfreiheit. Die Beschränkung ist aber nur unter gewissen Voraussetzungen zulässig (Art. $36 \mathrm{BV}$ ). Diese Voraussetzungen würden als allgemeine rechtsstaatliche Grundsätze auch dann gelten, wenn die Wirtschaftsfreiheit nicht zur Diskussion stünde (Art. 5 Abs. 1 und 2 BV).

\section{Einschränkung des Zugangs zur Berufs- ausübung im Rahmen der obligatorischen Krankenpflegeversicherung}

\section{Voraussetzungen}

Die in Art. 55a KVG und der Verordnung des Bundesrates vom 3. Juli 2002 vorgesehene befristete Bedürfnisklausel für Leistungserbringer im Rahmen der obligatorischen Krankenpflegeversicherung gemäss Art. 36-38 KVG ist zulässig, aber auch nur dann zulässig, wenn bestimmte
Voraussetzungen erfüllt sind. Es sind dies neben der gesetzlichen Grundlage - das öffentliche Interesse und die Verhältnismässigkeit der Massnahme (Art. 36 bzw. Art. 5 Abs. 1 und 2 BV). In der Beurteilung konkreter Fälle muss man dabei stets im Auge behalten, ob die Regelung, die unter diesen Gesichtspunkten überprüft wird, bereits in Art. 55a KVG enthalten oder dem Gestaltungsspielraum des Verordnungsgebers zuzurechnen ist. Die gesetzliche Regelung und eine Verordnungsbestimmung, welche deren zwingende Folge ist, ist durch Art. 191 BV in jedem Fall gedeckt; anderen Regelungen auf Verordnungsstufe hat der Richter die Anwendung $\mathrm{zu}$ versagen, wenn sie verfassungswidrig (oder gesetzwidrig) sind.

\section{Öffentliches Interesse}

Das Bestreben, den Kostenanstieg einzudämmen, um die soziale Krankenversicherung funktionsfähig zu erhalten, ist ein sozialpolitisches Motiv. Es rechtfertigt grundsätzlich Einschränkungen zu Lasten betroffener Privater. Ausnahmsweise kann selbst eine sozialpolitisch begründete Bedürfnisklausel - obwohl eine ausserordentlich einschneidende Massnahme - in Frage kommen. Bei dieser Betrachtungsweise verschiebt sich die Problematik der Bedürfnisklausel auf die Frage nach ihrer Verhältnismässigkeit.

\section{Verhältnismässigkeit}

Die Verhältnismässigkeit einer einschränkenden Massnahme wird unter den Aspekten der Geeignetheit, der Notwendigkeit, des Verbots des Übermasses und des Verbots des Missverhältnisses geprüft.

Ob eine Bedürfnisklausel für neue Kassenärzte geeignet ist, die Kosten im Gesundheitswesen zu senken, wird unterschiedlich beurteilt. In dieser Frage wird man daher der anordnenden Behörde einen erheblichen Beurteilungsspielraum zubilligen müssen. Im vorliegenden $\mathrm{Zu}$ sammenhang geht es allerdings nicht um eine auf Dauer angelegte, nachhaltige Bedürfnisklausel, sondern um eine auf höchstens drei Jahre befristete Massnahme. Als vorübergehender Stopp dient die Massnahme dem öffentlichen Interesse nur sehr beschränkt. Dazu kommt, dass Art. 55a KVG und die Verordnung des Bundesrates vom 3. Juli 2002 auf eine Art und Weise eingeführt wurden, welche die Wirksamkeit der befristeten Bedürfnisklausel in einem substantiellen Ausmass zunichte gemacht hat. Dies gilt vor allem in bezug auf die Verordnung, die hinsichtlich des Zeitpunktes ihres Erlasses und ihrer näheren Ausgestaltung nicht 
den Schutz von Art. 191 BV geniesst. Anstatt das Geschäft rasch durchzuziehen, wurde durch Orientierung der Öffentlichkeit einer Mehrzahl der Betroffenen Zeit gegeben, rechtzeitig vor Erlass der Verordnung ein Gesuch um Bewilligung zur Berufsausübung zu stellen (mit der gesetzlichen Folge der automatischen Zulassung zur Tätigkeit zu Lasten der obligatorischen Krankenpflegeversicherung). Davon wurde reichlich Gebrauch gemacht. Die Verordnung ist daher nur noch in Einzelfällen - und damit unter dem Aspekt der rechtsgleichen Behandlung in fragwürdiger Weise - geeignet, ihren Zweck zu erreichen.

Die Notwendigkeit (Erforderlichkeit) der Massnahme im jetzigen Zeitpunkt ist zweifelhaft, vor allem in Verbindung mit der Überlegung, ob nicht mildere Vorkehren gegen die Kostensteigerung genügen würden (Verbot des Übermasses). Als alternative und weniger einschneidende Massnahmen, die kurzfristig ergriffen werden könnten und sofort wirksam wären, kommen in Frage: Einschränkung des Leistungskatalogs in der obligatorischen Krankenpflegeversicherung (Art. 33 Abs. 1 KVG; Art. 12 Abs. 2 KVG betreffend Zusatzversicherungen setzt voraus, dass nicht alle Leistungen durch die soziale Krankenversicherung gedeckt sein müssen); höhere Kostenbeteiligung der Versicherten (Art. 64 Abs. 1 und Art. 6 Bst. a KVG); Verwirklichung - wenn schon - einer weniger starren Bedürfnisklausel (nicht völliger Ausschluss eines Arztes, sondern nur höhere Kostenbeteiligung des Versicherten); Ausschluss von Leistungserbringern, namentlich bei Verstössen gegen die Anforderungen der Wirtschaftlichkeit und der Qualitätskontrolle gemäss Art. 56 und 58 KVG. Allerdings wären die Auswirkungen dieser kurzfristig anzuordnenden Massnahmen vermutlich zu schwach, weshalb nicht eindeutig gesagt werden kann, die Anforderungen von Notwendigkeit und Übermassverbot seien für die kurzfristig angeordnete befristete Bedürfnisklausel nicht erfüllt.

Hingegen verletzt die Verordnung vom 3. Juli 2002 - unter Berücksichtigung der Art und Weise ihres Erlasses und ihrer Inkraftsetzung jedenfalls in einem Teil der Fälle das Verhältnismässigkeitsprinzip in seiner Ausprägung als Verbot des Missverhältnisses zwischen der im öffentlichen Interesse liegenden Kostenersparnis in der obligatorischen Krankenpflegeversicherung einerseits und den negativen Auswirkungen auf die betroffenen Ärzte anderseits. Der praktische Nutzen der nur kurzfristig wirksamen Verordnung ist gering, weil der Bundesrat durch die Art und Weise, wie die Verordnung erlassen wurde, es den meisten Interessenten, die in den nächsten drei Jahren eine Arztpraxis eröffnen wollen, ermöglicht hat, durch rechtzeitige Einreichung des Gesuches der Zulassungsbeschränkung auszuweichen (Art. 5 der Verordnung). In den Einzelfällen, in denen sich die Verordnung noch auswirkt, verbleibt anderseits die schwerwiegende Massnahme eines - wenn auch befristeten - Verbots, den Beruf in der gewünschten Art und Weise auszuüben.

In bezug auf Schweizer Bürger, die vor einer Praxiseröffnung im zeitlichen Geltungsbereich der Verordnung stehen, wirkt sich die Einführung der Bedürfnisklausel überdies als unzulässige unechte Rückwirkung der Verordnung aus. Diese Ärzte haben im Vertrauen darauf studiert, den Arztberuf selbständig ausüben und damit von Gesetzes wegen auch im Rahmen der sozialen Krankenpflegeversicherung tätig werden zu können. Auf sie darf die Verordnung schon unter diesem Gesichtspunkt nicht angewendet werden. Ausländische Ärzte konnten hingegen nicht damit rechnen, in der Schweiz den Arztberuf selbständig ausüben zu können. Dies gilt auch für Anwärter aus EU-Staaten. Unter dem Gesichtswinkel des Vertrauensschutzes wäre daher eine ungleiche Praxis - Nichtanwendung der Verordnung auf Schweizer Bürger, jedoch Anwendung auf Angehörige von EU-Staaten keine im Sinn von Art. 2 des Freizügigkeitsabkommens verpönte Diskriminierung [4].

\section{Ausgestaltung der Bedürfnisklausel}

\section{Verhältnis von Gesetz und Verordnung}

Art. 55a KVG ist inhaltlich sehr knapp gehalten. Er trifft materiell nur drei Regelungen: 1. Ermächtigung an den Bundesrat zur Einführung der Bedürfnisklausel für eine befristete Zeit von bis zu drei Jahren; 2. Auftrag an den Bundesrat, die entsprechenden Kriterien festzulegen; 3. Zuständigkeit der Kantone, die Leistungserbringer, die unter die Regelung fallen, zu bestimmen. Diese Regelungen sind im Sinn von Art. 191 BV von der Praxis unbedingt zu respektieren.

Der Bundesrat hat die Ausführungsvorschriften gesetzes- und verfassungskonform zu erlassen. Seine Regelungen sind für die Rechtsanwendung aber nur massgebend, soweit sie sich zwingend aus den inhaltlichen Vorgaben von Art. 55a KVG ergeben. Soweit sie im Rahmen des Gestaltungsspielraums des Bundesrates ergehen und gesetz- oder verfassungswidrig sein sollten, hat ihnen jedenfalls der Richter die Anwendung zu versagen. 


\section{Adressatenkreis}

Einbezug nur der frei praktizierenden Ärzte

Die Leistungserbringer im Rahmen der obligatorischen Krankenpflegeversicherung sind gleich zu behandeln (Art. 8 Abs. 1 BV). Der Gleichbehandlungsgrundsatz gilt qualifiziert hinsichtlich von Personen, die in Konkurrenz zueinander tätig sind; er ist hier auch eine Forderung der Wirtschaftsfreiheit. Art. 55a KVG sieht nur eine Bedürfnisklausel für die Zulassung von Leistungserbringern nach den Art. 36-38 KVG vor. Damit werden die Spitäler und andere Einrichtungen sowie Heilbäder, die in Art. 39 und $40 \mathrm{KVG}$ geregelt sind, nicht erfasst. Aus Art. 1 der (unsorgfältig redigierten) Verordnung vom 3. Juli 2002 ist dies zwar nicht ersichtlich, wohl aber aus den Anhängen. Die Begünstigung der Spitäler und ihrer als Angestellte praktizierenden Ärzte widerspricht krass dem Grundsatz der Gleichbehandlung der Wettbewerbsteilnehmer. Sie ist aber von Art. 55a KVG vorgezeichnet und damit für die Praxis tabu.

\section{Regelung zu Lasten der jungen Ärzte}

Art. 8 Abs. 2 BV bestimmt, dass niemand wegen des Alters diskriminiert werden darf. Eine unterschiedliche Behandlung ist jedoch zulässig, wenn hierfür qualifizierte Gründe vorliegen. Die Verordnung vom 3. Juli 2002 trifft im wesentlichen junge Ärzte. Die Beschränkung auf neue Gesuchsteller ist sachlich begründet. Die zusätzliche Einführung einer Altersgrenze wäre zwar ein naheliegendes Pendant, doch wäre die Einführung einer solchen Regelung ohne angemessene Übergangsfrist eine unzulässige unechte Rückwirkung und damit willkürlich. Art. 55a KVG sieht aber nur eine auf höchstens drei Jahre befristete Bedürfnisklausel vor.

\section{Gleichbehandlung in den Kantonen}

Als Sozialversicherungssystem des Bundes ist die obligatorische Krankenpflegeversicherung über das ganze Land hinweg nach einheitlichen Kriterien $\mathrm{zu}$ gestalten. Dieser Anforderung entspricht 55a Abs. $1 \mathrm{KVG}$, wonach der Bundesrat die Kriterien für die Zulassungsbeschränkungen erlässt. Die Regelung von Absatz 3, wonach die Kantone die Leistungserbringer nach Absatz 1 bestimmen, bezieht sich auf den Vollzug im Einzelfall und allenfalls noch auf die Festlegung von Kategorien nach Massgabe der Kriterien des Bundesrates im Sinn einer Berücksichtigung der unterschiedlichen Verhältnisse in den Kantonen. Die Bestimmung von Art. 2 Abs. 1 Buchstabe a der Verordnung, wonach die Kantone vorsehen können, dass die in Art. 1 (bzw. Anhang 1) festgelegte Höchstzahl für eine oder mehrere Kategorien von Leistungserbringern nicht gilt, hebt - wörtlich genommen, wie dies vom Bundesamt für Sozialversicherung in der Kommentierung vertreten wurde - die in Art. 55a Abs. $1 \mathrm{KVG}$ verlangte bundesrätliche Regelung aus den Angeln. Art. 2 Abs. 1 Buchstabe a der Verordnung ist somit gesetzwidrig.

\section{Kriterien der Zulassungsbeschränkungen}

Art. 55a Abs. 1 KVG bestimmt, dass der Bundesrat die Kriterien für die Feststellung des Bedürfnisses als Voraussetzung der Zulassung von Leistungserbringern zu Lasten der obligatorischen Krankenpflegeversicherung nach den Art. 36-38 KVG festlegt. Unter Kriterien sind Massstäbe zu verstehen, nach denen das Bedürfnis definiert wird. Der Bundesrat hat indessen weder festgelegt, unter welchen Voraussetzungen die $\mathrm{Zu}$ lassungsbeschränkungen grundsätzlich gelten sollen, noch nach welchen Grundsätzen das Bedürfnis für die einzelnen Kategorien von Leistungserbringern festzustellen ist. Der Bundesrat führt im Anhang 1 zur Verordnung lediglich Höchstzahlen auf, wobei aber nicht angegeben wird, nach welchen Kriterien diese Höchstzahlen festgelegt sind. Dieses unübersichtliche Regime wird überdies durch die Ermächtigung an die Kantone relativiert und völlig undurchschaubar gemacht, Höchstzahlen als nicht geltend zu bezeichnen (Art. 2 Abs. 1 Bst. a der Verordnung) und bei Unterversorgung (nach welchen Kriterien festgestellt?) zusätzliche Leistungserbringer zuzulassen (Art. 3 der Verordnung). Damit wird die Vorgabe von Art. 55a Abs. 1 KVG, wonach der Bundesrat die Kriterien festlegt, krass missachtet. Ein Arzt, der nicht zur Tätigkeit zu Lasten der obligatorischen Krankenpflegeversicherung zugelassen wird, kann daher geltend machen, die Verordnung setze Art. 55a KVG nicht gesetzeskonform um. Es fehlt zurzeit eine Verordnungsgrundlage, welche die gesetzeskonforme Durchführung der in Art. 55a KVG vorgesehenen Bedürfnisklausel ermöglichen würde. 